\title{
Finite difference implicit structural modeling of geological structures
}

\author{
Modeste Irakarama • Gautier Laurent · \\ Julien Renaudeau • Guillaume Caumon
}

Received: 15 July 2019 / Accepted: 10 Aout 2020 / Published Online 15 Sept 2020

\begin{abstract}
We introduce a new method for implicit structural modeling. The main developments in this paper are the new regularization operators we propose by extending inherent properties of the classic one-dimensional discrete second derivative operator to higher dimensions. The proposed regularization operators discretize naturally on the Cartesian grid using finite differences, owing to the highly symmetric nature of the Cartesian grid. Furthermore, the proposed regularization operators do not require any special treatment on boundary nodes, and their generalization to higher dimensions is straightforward. As a result, the proposed method has the advantage of being simple to implement. Numerical examples show that the proposed method is robust and numerically efficient.
\end{abstract}

Keywords Implicit modeling · structural modeling · regularization operators · finite-differences $\cdot$ interpolation

Author's version, Copyright IAMG and Springer Nature. Published as doi:10.1007/s11004-02009887-w

Modeste Irakarama

Université de Lorraine, CNRS, GeoRessources lab., 2 rue du Doyen Marcel Roubault, Vandœuvre-lès-Nancy, F-54518, France

E-mail: modeste.irakarama@univ-lorraine.fr

Gautier Laurent

Université d'Orléans,CNRS, BRGM, ISTO, UMR 7327, F-45071, Orléans, France

E-mail: gautier.laurent@univ-orleans.fr

Julien Renaudeau

Université de Lorraine, CNRS, GeoRessources lab., 2 rue du Doyen Marcel Roubault, Vandœuvre-lès-Nancy, F-54518, France

E-mail: julien.renaudeau@univ-lorraine.fr

Guillaume Caumon

Université de Lorraine, CNRS, GeoRessources lab., 2 rue du Doyen Marcel Roubault, Vandœuvre-lès-Nancy, F-54518, France

E-mail: guillaume.caumon@univ-lorraine.fr 


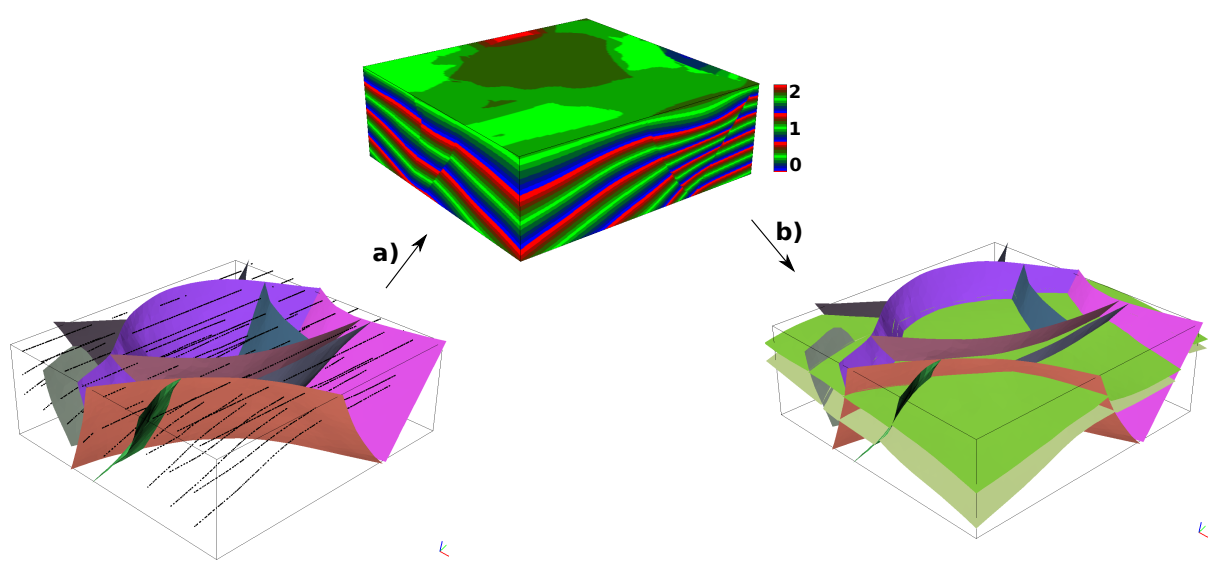

Fig. 1 Implicit structural modeling workflow. a) Interpolation of input data to build a stratigraphic function using the proposed method. b) Extraction of implicit horizons from the isovalues of the stratigraphic function. Data courtesy of Total.

\section{Introduction}

Structural implicit modeling, in this paper, is defined as the interpolation of randomly distributed, and possibly sparse, structural data. For simplicity, structural data, also referred to as structural constraints, will be limited to the following:

$$
\begin{gathered}
\text { value constraints : } \phi\left(\mathbf{x}_{j}\right)=a_{j} \\
\text { orientation constraints : } \nabla \phi\left(\mathbf{x}_{j}\right)=\left\|\nabla \phi\left(\mathbf{x}_{j}\right)\right\| \mathbf{u}_{j} ;
\end{gathered}
$$

where $\phi(\mathbf{x})$ is the unknown function to be interpolated, $\mathbf{x}$ is a point in space, $a$ is some given scalar, and $\mathbf{u}$ is some given unit vector. We refer to Eq. (2) as the normal form of an orientation constraint, which informs both about the direction and the norm of $\phi$, as opposed to the tangential form, which informs only about the orientation of $\phi$. Let $\mathbf{u}^{1}$ be a given unit vector, then there are $N-1$ unit vectors $\left\{\mathbf{u}^{l}\right\}_{l=2}^{l=N}$ such that $\left\{\mathbf{u}^{l}\right\}_{l=1}^{l=N}$ forms an orthogonal basis in $N \geq 2$ dimensions. In that case, the tangential form of the normal orientation constraint $\nabla \phi(\mathbf{x})=\|\nabla \phi(\mathbf{x})\| \mathbf{u}^{1}$ is

$$
\mathbf{u}^{l} \cdot \nabla \phi(\mathbf{x})=0, \text { for } l=2, \ldots, N .
$$

In implicit structural modeling, the object being modeled is obtained by extracting a hypersurface along an iso-value of the interpolated function $\phi(\mathbf{x})$ (see Newman and Yi, 2006, for a survey on this topic). This principle can be distinguished from explicit structural modeling, where geological interfaces defined by a twodimensional planar graph are embedded in three-dimensional space (see for example Caumon et al., 2009).

Implicit structural modeling has extensively been used in geosciences for contour mapping (Briggs, 1974, Mallet, 1984, Smith and Wessel, 1990, Wessel and Bercovici, 1998), and for three-dimensional geological modeling (Lajaunie et al., 1997, Mallet, 2004). The method presented here was developed mainly for geological modeling applications, but it is suited for contour mapping as well. Most of the 
groundwork of modern structural implicit modeling were developed in the 2000's (Cowan et al., 2002, Ledez, 2003, Chilès et al., 2004, Moyen, 2005, Tertois, 2007), starting with the pioneering work of Lajaunie et al. (1997), to the theoretical framework developed by Mallet (2004), all the way to the more application-ready works of Chilès et al. (2004) and Frank (2007). Structural implicit modeling can be separated into two main classes (Caumon et al., 2013): (1) methods based on dual Kriging and radial basis interpolation (Lajaunie et al., 1997; Cowan et al., 2002, Chilès et al., 2004, Calcagno et al., 2008), which yield a dense linear system whose size is mainly controlled by the number of data, (2) and methods based on domain discretization (Mallet, 2004; Frank, 2007; Caumon et al., 2013, Souche et al., 2013), which yield a sparse linear system whose size is mainly controlled by size of discretization grid. Domain, in this paper, refers to the volume of interest under investigation. Domain discretization methods are collectively referred to as Discrete Smooth Interpolation (DSI) methods hereafter (Mallet, 1989, 1992, 1997). The principle of DSI methods is to discretize all structural constraints on a discrete domain, and assemble them into a least-squares system of linear equations supplemented with smoothing regularization constraints (Mallet, 1989, 1992, 1997).

Implicit geological modeling is still an active area of research. Some authors have shown that existing methods still present limitations caused by the smoothing approaches and the discretization schemes currently used (Laurent, 2016, Renaudeau et al., 2017b). In addition, recent work started investigating stochastic modeling approaches of geological structures geometry (Jessell et al., 2014, Cherpeau and Caumon, 2015, Godefroy et al., 2017) for which structural interpolation is becoming a bottleneck. Computation times that used to be acceptable for building a single "best model" are becoming far too long when considering stochastic approaches for sampling uncertainty. Other recent research and advances in implicit modeling include better modeling of folds (Laurent et al., 2016 , Grose et al., 2017), automated building of models that conform to seismic data (Wu, 2017), more numerically efficient discretization schemes (Renaudeau et al. 2018), and many more (Mallet, 2014, Hillier et al., 2014, Gonçalves et al., 2017, Martin and Boisvert, 2017, Renaudeau et al., 2019). As we move towards an era of multi-realization structural modeling (Caumon, 2010), new challenges continuously emerge and motivate the quest for more robust and more efficient structural implicit modeling schemes.

In this paper, we introduce a new approach based on finite differences; this method belongs to the DSI class of methods. Most DSI methods, as applied in geological modeling, rely on simplices (triangles in two-dimensions, tetrahedra in three-dimensions) for domain discretization due to their geometrical flexibility. Because simplicial meshes are unstructured and irregular, it is customary to discretize structural constraints and smoothing constraints using a piecewise linear approach (Frank, 2007, Caumon et al., 2013). The method we introduce is based on discretizing the domain on Cartesian grids, and on discretizing structural constraints and smoothing constraints using finite differences. Cartesian gridding and finite differences are arguably the simplest discretizations possible for structural interpolation; as a result, the proposed method is also easy to implement. Modern computing capabilities, particularly general purpose GPU computing, make 
a)

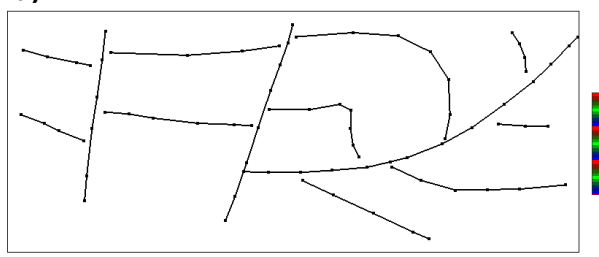

b)

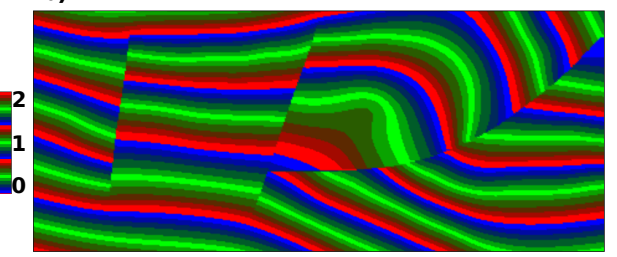

Fig. 2 Implicit stratigraphic modeling of a section from the Ribaute model of Caumon et al. (2009). a) Input data, three faults and three horizons. b) Resulting stratigraphic field. Only value constraints were used in this experiment.

the method numerically efficient as well. A three-dimensional implicit structural modeling example using the proposed method is shown in Fig. 1.

Implicit structural interpolation on Cartesian grids using finite differences is not new. Briggs (1974) solves the biharmonic equation in two-dimensions directly on the Carstesian grid using finite differences. In Briggs (1974)'s formulation, assignment constraints (Eq. (1)) are treated as internal boundary conditions. He also shows the equivalence between the biharmonic equation and the minimization of the global curvature of the implicit function. Wu and Hale (2015) use finite differences operators on Cartesian grids to create implicit functions on seismic images. Assuming that (1) the normal of reflectors can be estimated at each point of the seismic image, and that (2) the implicit function increases monotonically with seismic traveltime (or depth), they use the normal form of orientation constraints (Eq. (2)) to assemble an overdetermined system solved using least-squares. However, it is usually challenging to make an accurate estimation of reflector dips at each grid point due to coherent noise in seismic data (Chauris et al., 2002; Fehmers and Höcker, 2003). As for the second assumption, it is not always valid; the section from the Ribaute model (Caumon et al., 2009) in Fig. 2 is an example of where this assumption does not hold. $\mathrm{Wu}(2017)$ addresses this problem by first unfaulting and unfolding the seismic image before computing the implicit function. Mallet (1989) also proposed an interpolation method on Cartesian grids using finite differences; the method proposed here resolves some issues raised by Mallet (1989), as discussed in the section about discontinuities (Sect.6). Furthermore, unlike Mallet (1989), the method proposed here does not require input data to be located at grid points.

We propose an alternative approach. Briggs (1974) noted that the one-dimensional interpolation problem had well defined properties; his strategy was then to extend these properties in two-dimensions by solving the equivalent continuous problem (partial differential equation). Here, we notice that the one-dimensional discrete interpolation problem has inherent properties that we wish to extend to high dimensional discrete interpolation problems. The main new elements of our method are the regularization operators we introduce. These operators discretize naturally on Cartesian grids: they do not require any special treatment at boundary nodes, and their generalization to higher dimensions is straightforward. 
a)

b)

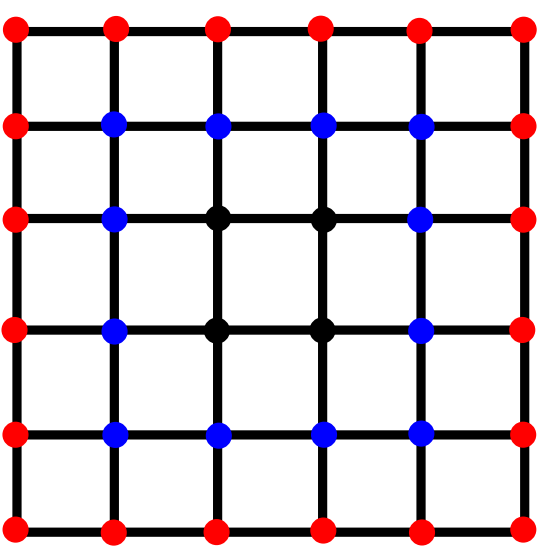

Fig. 3 a) One-dimensional domain discretization. b) Two-dimensional domain discretization. The red nodes are external nodes, the blue nodes are boundary nodes, and the black nodes are internal nodes.

This paper is organized as follows. In Sects. 2 and 3 we present the basic theoretical aspects of our method. We apply our method in two-dimensions in Sect. 4. and then in three-dimensions in Sect.5. We discuss how to handle discontinuities in Sect. 6 before concluding with a discussion on the shortcomings of the proposed method in Sect. 7 .

\section{Domain and problem discretization}

\subsection{Domain discretization}

Let $\Omega$ be the domain of definition of $\phi(\mathbf{x}) . \Omega$ is discretized on a Cartesian grid, therefore $\Omega$ can be seen as a collection of $N$ grid points $\left\{\mathbf{x}_{l}\right\}_{l=1}^{l=N}$. We distinguish three subdomains in $\Omega$,

$$
\Omega=\left\{\Omega_{E} \cup \Omega_{B} \cup \Omega_{I}\right\},
$$

where $\Omega_{E}$ is the set of external grid points, $\Omega_{B}$ is the set of boundary grid points, and $\Omega_{I}$ is the set of internal grid points. An example of this discretization is illustrated in Fig. 3. A more formal definition of these subdomains will be given shortly. The computational domain $\Omega_{C}$ is defined as

$$
\Omega_{C}=\left\{\Omega_{B} \cup \Omega_{I}\right\}
$$

Each point in $\mathbf{x}_{j}$ in $\Omega_{I}$ has neighbors in $\Omega_{C}$, these neighbors define the neighborhood $\mathcal{N}\left(\mathbf{x}_{j}\right)$ of $\mathbf{x}_{j}$. Figures $4 \mathrm{a}$ and $4 \mathrm{p}$ illustrate the notion of neighborhoods in one and two-dimensions; this figure also introduces directional vectors. For example, the one-dimensional point $x_{j}$ has two neighbors

$$
\mathcal{N}\left(x_{j}\right)=\left\{x_{j-1}, x_{j+1}\right\}:=\left\{x_{j-d_{1}}, x_{j+d_{1}}\right\}
$$


b)

a)
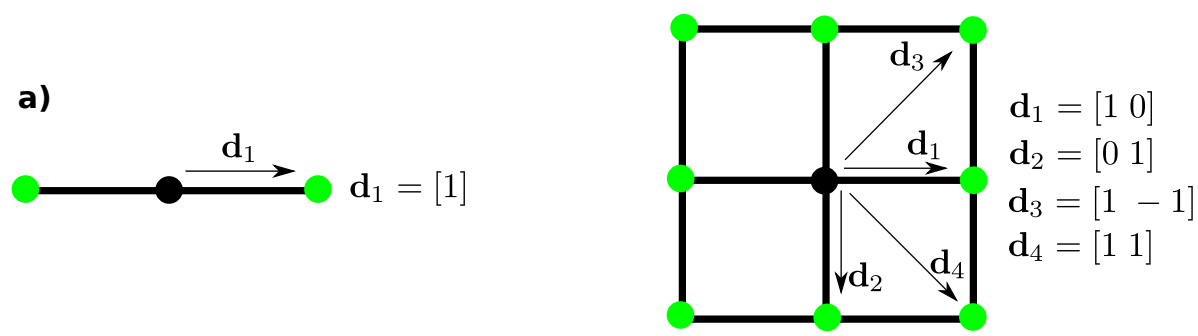

Fig. 4 Notion of neighborhoods and directional vectors in one and two-dimensions. a) Onedimensional neighborhood: the neighborhood of the black node is made of the two (green) points that can be reached from it by taking one step (one forward and one backward) along the one-dimensional directional vector. b) Two-dimensional neighborhood: the neighborhood of the black node is made of the eight (green) points that can be reached from it by taking one step (one forward and one backward) along the four two-dimensional directional vectors.

that is, by definition, the one-dimensional directional vector $d_{1}$ is [1]. In general, a point $\mathbf{x}_{j}$ always has two neighbors along a given direction: one neighbor in front, and one neighbor behind. For example in two-dimensions the two neighbors of the point $\mathbf{x}_{i, j}=\mathbf{x}_{k}$ along the direction $\mathbf{d}_{4}=\left[\begin{array}{ll}1 & 1\end{array}\right]$ are

$$
\left\{\mathbf{x}_{i-1, j-1}, \mathbf{x}_{i+1, j+1}\right\}:=\left\{\mathbf{x}_{k-\mathbf{d}_{4}}, \mathbf{x}_{k+\mathbf{d}_{4}}\right\} .
$$

In $N$ dimensions, there are $M_{n}=3^{N}-1$ points in the neighborhood $\mathcal{N}\left(\mathbf{x}_{j}\right)$ of $\mathbf{x}_{j} \in \Omega_{I}$, and there are $M_{d}=\frac{M_{n}}{2}$ directions such that

$$
\mathcal{N}\left(\mathbf{x}_{j}\right)=\left\{\mathbf{x}_{j \pm \mathbf{d}_{k}} \mid \text { for } k=1, \ldots, M_{d}\right\}
$$

where $\mathbf{d}_{k}$ is the $k^{t h}$ directional vector, as illustrated for example in Fig. 4

Let us come back to the discretization $\Omega=\left\{\Omega_{E} \cup \Omega_{B} \cup \Omega_{I}\right\}$. Given a set of points in $\Omega_{E}$, we formally define

$$
\begin{aligned}
\Omega_{I} & =\left\{\mathbf{x}_{j} \mid \mathcal{N}\left(\mathbf{x}_{j}\right) \cap \Omega_{E}=\emptyset\right\} . \\
\Omega_{B} & =\left\{\mathbf{x}_{j} \mid \mathcal{N}\left(\mathbf{x}_{j}\right) \cap \Omega_{E} \neq \emptyset \wedge \mathcal{N}\left(\mathbf{x}_{j}\right) \cap \Omega_{I} \neq \emptyset\right\} .
\end{aligned}
$$

That is, a grid point that does not have a neighbor in $\Omega_{E}$ is by definition a point in $\Omega_{I}$, and a grid point that has at least one neighbor in $\Omega_{I}$ and at least one neighbor in $\Omega_{E}$ is by definition a point in $\Omega_{B}$. Points in $\Omega_{E}$ will usually be specified as inputs. It should be noted that an internal grid point is never in contact with an external grid point, there is always at least one boundary point between them.

\subsection{Problem discretization}

The implicit function $\phi(\mathbf{x})$ is evaluated on the discrete computational domain $\Omega_{C}$ by expansion into the series

$$
\phi(\mathbf{x})=\sum_{\mathbf{x}_{j} \in \Omega_{C}} B\left(\mathbf{x}, \mathbf{x}_{j}\right) \phi\left(\mathbf{x}_{j}\right)=\sum_{\mathbf{x}_{j} \in \Omega_{C}} B\left(\mathbf{x}, \mathbf{x}_{j}\right) \phi_{j},
$$


where $B\left(\mathbf{x}, \mathbf{x}_{j}\right)$ are some basis functions, and $\phi_{j}$ are the unknown coefficients at grid points. The basis functions $B\left(\mathbf{x}, \mathbf{x}_{j}\right)$ are assumed to satisfy the Kronecker delta property, that is for $\mathbf{x}_{i}, \mathbf{x}_{j} \in \Omega_{C}$,

$$
B\left(\mathbf{x}_{i}, \mathbf{x}_{j}\right)=\left\{\begin{array}{l}
1 \text { if } i=j \\
0 \text { if } i \neq j
\end{array} .\right.
$$

Let $B\left(\mathbf{x}, \mathbf{x}_{j}\right)$ be $1^{\text {st }}$-order Lagrange basis functions, simplifying Eq. (9) to

$$
\phi(\mathbf{x})=\sum_{\mathbf{x}_{j} \in \mathcal{N}^{\prime}\left(\mathbf{x}_{i}\right)} B\left(\mathbf{x}, \mathbf{x}_{j}\right) \phi_{j},
$$

where $\mathbf{x}_{i} \in \Omega_{I}$ is the closest internal grid point to $\mathbf{x}$, and

$$
\mathcal{N}^{\prime}\left(\mathbf{x}_{i}\right):=\left\{\mathcal{N}\left(\mathbf{x}_{i}\right) \cup \mathbf{x}_{i}\right\}
$$

it follows from the definitions in Eqs. (7) and (8) that $\mathbf{x}_{j} \in \Omega_{C}$. Using Eq. (10), the first derivative along the $k^{t h}$ direction is given by

$$
\partial_{k} \phi(\mathbf{x})=\sum_{\mathbf{x}_{j} \in \mathcal{N}^{\prime}\left(\mathbf{x}_{i}\right)} \partial_{k} B\left(\mathbf{x}, \mathbf{x}_{j}\right) \phi_{j} .
$$

The constraints in Eqs. (1) and (3) can therefore be discretized in $N \geq 2$ dimensions as

$$
\phi(\mathbf{x})=\sum_{\mathbf{x}_{j} \in \mathcal{N}^{\prime}\left(\mathbf{x}_{i}\right)} B\left(\mathbf{x}, \mathbf{x}_{j}\right) \phi_{j}=a,
$$

and

$$
\begin{aligned}
\mathbf{u}^{l} \cdot \nabla \phi(\mathbf{x}) & =\sum_{k}^{N} u_{k}^{l} \partial_{k} \phi(\mathbf{x}) \\
& =\sum_{k}^{N} u_{k}^{l}\left(\sum_{\mathbf{x}_{j} \in \mathcal{N}^{\prime}\left(\mathbf{x}_{i}\right)} \partial_{k} B\left(\mathbf{x}, \mathbf{x}_{j}\right) \phi_{j}\right)=0, \text { for } l=2, \ldots, N
\end{aligned}
$$

Some constraints may involve second derivatives; this is typically the case for smoothing regularization constraints. However, second derivatives of Eq. (10) cannot be computed directly, as was the case for first derivatives, because of the use of $1^{\text {st }}$-order Lagrange basis functions. The second derivative at a given point $\mathbf{x}$ is therefore approximated by the finite-difference second derivative at $\mathbf{x}_{i} \in \Omega_{I}$, the closest internal grid point to $\mathbf{x}$. In particular, let $\mathrm{d}_{k}$ denote the distance between two neighboring points along the directional vector $\mathbf{d}_{k}$; then, using the notation in the definition in Eq. (5), the second derivative along the $k^{t h}$ direction is given by

$$
\partial_{k}^{2} \phi(\mathbf{x}) \approx \partial_{k}^{2} \phi\left(\mathbf{x}_{i}\right)=\frac{-2 \phi_{i}+\phi_{i+\mathbf{d}_{k}}+\phi_{i-\mathbf{d}_{k}}}{\mathrm{~d}_{k}^{2}}
$$

and the mixed derivative along two orthogonal directions $k, l$ is given by

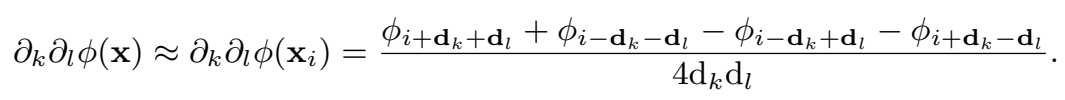

In this paper, all constraints involving second derivatives are imposed strictly on grid points; therefore, the approximation symbol $\approx$ in Eqs. 14 and 15 can be replaced by the equality symbol $=$. 
2.3 Solving the discrete problem

The system of Eqs. 12)- 13 , can be written in the more compact matrix form

$$
\begin{gathered}
\overline{\overline{\mathbf{A}}} \bar{\Phi}=\overline{\mathbf{f}}_{a} . \\
\overline{\mathbf{U}} \bar{\Phi}=\overline{\mathbf{0}} .
\end{gathered}
$$

In practice, the system above is usually underdetermined and we have to regularize it with some smoothing constraints of the form $\overline{\mathbf{R}} \bar{\Phi}=\overline{\mathbf{0}}$. That is, the solution $\bar{\Phi}$ is obtained by solving

$$
\left[\begin{array}{c}
\overline{\overline{\mathbf{A}}} \\
\overline{\mathbf{U}} \\
\overline{\overline{\mathbf{R}}}
\end{array}\right][\bar{\Phi}]=\left[\begin{array}{c}
\overline{\mathbf{f}_{a}} \\
\overline{\mathbf{0}} \\
\overline{\mathbf{0}}
\end{array}\right]
$$

in a least-squares sense. Convenient choices for $\overline{\overline{\mathbf{R}}}$ will be proposed later in the paper. The matrices involved in Eq. (18) are very sparse, making it possible to efficiently solve the least-squares system with sparse conjugate gradient solvers.

\section{Interpolation in one-dimension: problem formulation}

\subsection{Interpolation in one-dimension}

The structural interpolation problem can be described as a problem of finding a smooth function $\phi(\mathbf{x})$ that interpolates a given set of structural data. The smoothness is typically achieved by minimizing the function's roughness $\mathcal{R}(\phi)$ (Mallet, 1989). It is unclear what the definition of roughness is, precisely; roughness is usually understood to be related to the notion of curvature. In one-dimension, a widely accepted definition of roughness is the second derivative operator (Mallet, 1997)

$$
\mathcal{R}(\phi):=\partial_{x}^{2} \phi(\mathbf{x})
$$

Minimizing the second derivative in one-dimension minimizes the curvature of the function; this statement is unambiguous because there is only one way to define curvature in one-dimension. The one-dimensional smoothing constraint, defined only on grid points, can therefore be discretized using Eq. (14) to give

$$
\mathcal{R}\left(\phi_{j}\right)=\frac{-2 \phi_{j}+\phi_{j+1}+\phi_{j-1}}{|d x|^{2}}=0
$$

for every grid point $x_{j} \in \Omega_{I}$.

\subsection{Problem formulation}

In higher dimensions, minimizing the roughness of a function becomes ambiguous: there are infinitely many candidate roughness operators in higher dimensions that reduce to Eq. (19) in one-dimension. The standard way of overcoming this ambiguity is to explicitly give a physical meaning to Eq. (19). For example, Briggs (1974) and Levy (1999) explicitly look for a function $\phi(\mathbf{x})$ that minimizes the 
global (mean) curvature; in that case, it becomes clear that the extension of Eq. (19) in higher dimensions is

$$
\mathcal{R}(\phi)=\Delta \phi(\mathbf{x}),
$$

which is unambiguous since the Laplacian operator $\Delta$ has a precise definition in all dimensions. Another common choice is to seek for a function $\phi(\mathbf{x})$ that minimizes the bending energy (see for example Renaudeau et al., 2019, and references therein).

In this paper, we take a different approach to determine the equivalent of Eq. (19) in higher dimensions. We find inherent properties of the one-dimensional discrete Eq. 20 that we consider to be practical, from an implementation point of view, then we look for an equivalent discrete equation in higher dimensions which has the same properties. The resulting discrete operator in higher dimensions can then be transformed back to the continuous version if needed.

A very practical property of sparse data interpolation using Eq. (20) is that it does not require boundary conditions. We define a boundary condition as any constraint imposed on a grid point $\mathbf{x} \in \Omega_{B}$. In the absence of discontinuities, a regularization matrix $\overline{\overline{\mathbf{R}}}$ built by imposing Eq. 20 on all grid points $\mathbf{x} \in \Omega_{I}$, has a $\operatorname{rank}(\overline{\overline{\mathbf{R}}})=M-2$, for a one-dimensional problem with $M$ degrees of freedom (i.e. $M$ grid points in $\Omega_{C}$ ). The property $\operatorname{rank}(\overline{\overline{\mathbf{R}}})=M-2$ highlights the fact that a minimum of two independent points are required to define a function in one-dimension. In general, $\mathcal{R}\left(\phi_{j}\right)$ will be said to satisfy the maximum-rank property if, in the absence of discontinuities, $\operatorname{rank}(\overline{\overline{\mathbf{R}}})=M-(N+1)$; where $\overline{\overline{\mathbf{R}}}$ is the regularization matrix built by imposing $\mathcal{R}\left(\phi_{j}\right)=0$ for all grid points $\mathbf{x}_{j} \in \Omega_{I}$, for a problem with $M$ degrees of freedoms in $N$ dimensions. Our aim is to look for roughness operators $\mathcal{R}\left(\phi_{j}\right)$ that satisfy the maximum-rank property in higher dimensions.

The maximum-rank property can be extended to higher dimensions if the high dimensional equivalent of Eq. 20 has the following properties:

Property $1 \mathcal{R}\left(\phi_{j}\right)$ should include all grid points in the neighborhood of $\mathbf{x}_{j}$, and $\mathbf{x}_{j}$ itself.

Property $2 \mathcal{R}\left(\phi_{j}\right)$ should smooth independently along all directional vectors at $\mathbf{x}_{j}$.

The two properties above are inherent in the one-dimensional discrete problem, as it can be observed by looking at Eq. 20) and Fig. 4 a. Therefore, we propose to generalize these two properties to higher dimensions. As we increase dimensions, the first property will guarantee that every degree of freedom is taken into account, and the second property will guarantee that there are "enough" equations to constrain all the degrees of freedom.

\subsection{Towards interpolation in higher dimensions}

We propose two strategies for extending Eq. 20 to higher dimensions. The first one is to generalize the one-dimensional smoothing constraint

$$
\mathcal{R}\left(\phi_{j}\right)=\partial_{x}^{2} \phi_{j}=0
$$




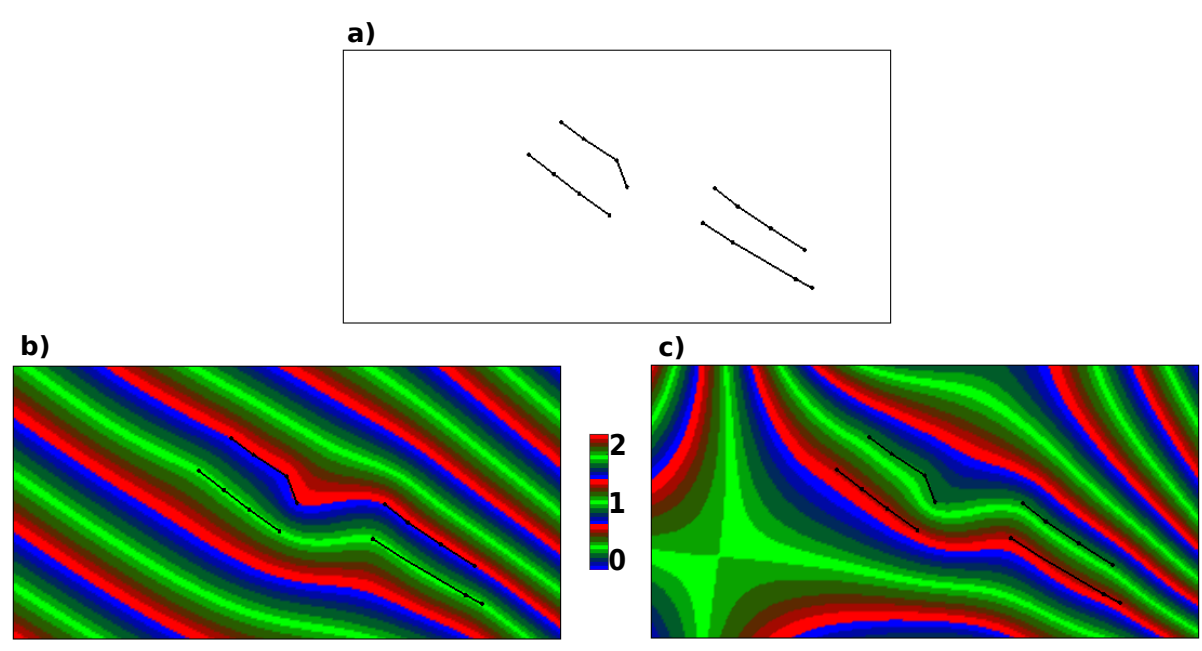

Fig. 5 Implicit stratigraphic modeling of a modified section from one of the two-dimensional benchmark models of Renaudeau et al. (2017a) a) Input data, two horizons. b) Resulting stratigraphic field using the proposed regularization operator. c) Resulting stratigraphic field using the Laplacian operator. Only value constraints were used in this experiment.

as a minimization of second directional derivatives of $\phi_{j}$ along all directional vectors, that is:

$$
\mathcal{R}\left(\phi_{j}\right)=\partial_{k}^{2} \phi_{j}=0, \forall \text { directional vectors } \mathbf{d}_{k} \text { at } \mathbf{x}_{j} .
$$

The second approach is to generalize the one-dimensional smoothing constraint

$$
\mathcal{R}\left(\phi_{j}\right)=\partial_{x}^{2} \phi_{j}=\partial_{x}\left(\partial_{x} \phi_{j}\right)=0
$$

as a minimization of mixed derivatives of $\phi_{j}$ as follows:

$$
\mathcal{R}\left(\phi_{j}\right)=\partial_{k}\left(\partial_{l} \phi_{j}\right)=0, \mid \begin{aligned}
& \forall \text { axis-aligned directional vectors } \mathbf{d}_{k}, \text { and } \\
& \forall \text { directional vectors } \mathbf{d}_{l} \text { s.t. } \mathbf{d}_{l}=\mathbf{d}_{k} \text { or } \mathbf{d}_{l} \perp \mathbf{d}_{k} .
\end{aligned}
$$

Both Eq. 22 and Eq. 23 satisfy the properties imposed in the last paragraph; these two equations lead to different roughness operators.

\section{Interpolation in two-dimensions}

Let us introduce the two-dimensional Cartesian-axes matrix $\overline{\overline{\mathbf{C}}}_{2}$ and the twodimensional diagonal-axes matrix $\overline{\overline{\mathbf{D}}}_{2}$ defined as

$$
\overline{\overline{\mathbf{C}}}_{2}=\left[\begin{array}{ll}
1 & 0 \\
0 & 1
\end{array}\right], \quad \overline{\overline{\mathbf{D}}}_{2}=\frac{1}{\sqrt{2}}\left[\begin{array}{cc}
1 & -1 \\
1 & 1
\end{array}\right] .
$$

Each row of these matrices is a directional vector on the two-dimensional grid as defined in Fig. 4. b. The two-dimensional full direction matrix is defined as

$$
\overline{\overline{\mathbf{F}}}_{2}=\left[\begin{array}{l}
\overline{\overline{\mathbf{C}}}_{2} \\
\overline{\overline{\mathbf{D}}}_{2}
\end{array}\right],
$$

its rows give all the directions available, locally, on a two-dimensional grid. 
4.1 Directional second derivatives

From the directional second derivative point of view (Eq. (22)), the two-dimension version of Eq. 20 is

$$
\mathcal{R}\left(\phi_{j}\right)=\partial_{k}^{2} \phi_{j}=\frac{-2 \phi_{j}+\phi_{j+\mathbf{d}_{k}}+\phi_{j-\mathbf{d}_{k}}}{\mathrm{~d}_{k}^{2}}=0, \forall \mathbf{d}_{k} \text { rows of } \overline{\overline{\mathbf{F}}}_{2}
$$

Each of these directional derivatives $\partial_{k}^{2} \phi_{j}$ can be written analytically using the two-dimensional Hessian matrix

$$
\overline{\overline{\mathbf{H}}}_{2}=\left[\begin{array}{ll}
\partial_{x}\left(\partial_{x} \phi\right) & \partial_{x}\left(\partial_{z} \phi\right) \\
\partial_{z}\left(\partial_{x} \phi\right) & \partial_{z}\left(\partial_{z} \phi\right)
\end{array}\right]
$$

as $\partial_{k}^{2} \phi=\mathbf{d}_{k} \overline{\overline{\mathbf{H}}}_{2} \mathbf{d}_{k}^{T}$. It follows that a two-dimension version of the roughness operator 19] that satisfies the maximum-rank property is

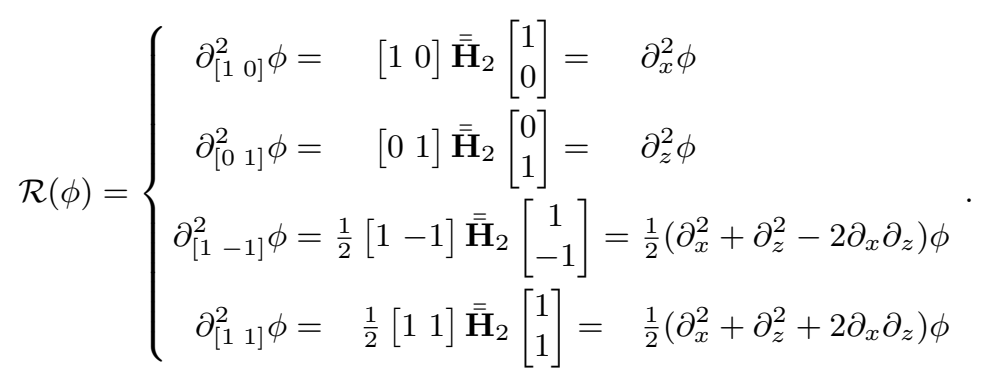

The roughness operator (27) is discretized exactly as the one-dimensional version. Let $\overline{\overline{\mathbf{R}}}$ denote the regularization matrix resulting from Eq. $(26) /(27)$, then the $j^{t h}$ row of the least-squares matrix $\overline{\overline{\mathbf{R}}}^{t} \overline{\overline{\mathbf{R}}}$ is a finite-difference approximation of

$$
\left.\left[\left(\partial_{\left[\begin{array}{ll}
1 & 0
\end{array}\right]}^{2}\right)^{2}+\left(\partial_{[0}^{2} 1\right]\right)^{2}+\left(\partial_{[1-1]}^{2}\right)^{2}+\left(\partial_{\left[\begin{array}{ll}
1 & 1
\end{array}\right]}^{2}\right)^{2}\right] \phi_{j}
$$

It is interesting to note that Eq. (28) equates to

$$
\left[\left(\partial_{x}^{2}\right)^{2}+\left(\partial_{z}^{2}\right)^{2}+2\left(\partial_{x} \partial_{z}\right)^{2}\right] \phi_{j}+\frac{1}{2}\left[\left(\partial_{x}^{2}\right)^{2}+\left(\partial_{z}^{2}\right)^{2}+2 \partial_{x}^{2} \partial_{z}^{2}\right] \phi_{j}
$$

where the first term is the bending energy of Enriquez et al. (1983); Turk and O'Brien (2002); Renaudeau et al. (2019), and the second term is half the squared (mean) curvature of Briggs (1974), also known as the squared Laplacian (Levy, 1999). Figure 5b shows an example using the roughness operator $(26) /(27)$ to a modified version of a two-dimensional benchmark model proposed by Renaudeau et al. (2017a). For comparison, Fig. 5s shows the implicit function obtained using the Laplacian operator (Eq. 21) ; note the saddle point in Fig. 56, which is physically impossible from a stratigraphic modeling point of view. 
4.2 Mixed derivatives

From the mixed derivative point of view (Eq. $(23)$ ), another roughness operator $\mathcal{R}(\phi)$ that satisfies the maximum-rank property is

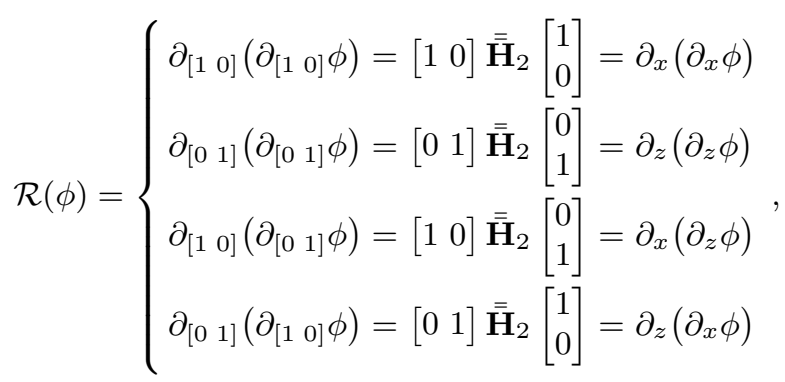

which, using $\partial_{x}\left(\partial_{z}\right)=\partial_{z}\left(\partial_{x}\right)$, becomes

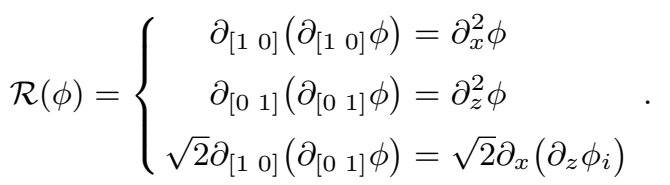

The factor $\sqrt{2}$ comes from noticing that

$$
\left(\partial_{\left[\begin{array}{ll}
1 & 0
\end{array}\right]}\left(\partial_{\left.\left[\begin{array}{ll}
0 & 1
\end{array}\right]\right)}\right)\right)^{2} \phi_{j}+\left(\partial_{\left[\begin{array}{ll}
0 & 1
\end{array}\right]}\left(\partial_{\left[\begin{array}{ll}
1 & 0
\end{array}\right]}\right)\right)^{2} \phi_{j}=\left(\sqrt{2} \partial_{\left[\begin{array}{ll}
1 & 0
\end{array}\right]}\left(\partial_{\left[\begin{array}{ll}
0 & 1
\end{array}\right]}\right)\right)^{2} \phi_{j}
$$

as in the previous paragraph, the reason for equating squares of operators is because the problem is solved by least-squares. The complete sum of squares of terms in this operator equates to the first term on the right hand side of Eq. (29). The first two terms of Eq. (30) are discretized using Eq. (14) and the remaining terms are discretized using Eq. [15.

\section{Interpolation in three-dimensions}

The three-dimensional Cartesian-axes matrix $\overline{\overline{\mathbf{C}}}_{3}$ and diagonal-axes matrix $\overline{\overline{\mathbf{D}}}_{3}$ are

$$
\overline{\overline{\mathbf{C}}}_{3}=\left[\begin{array}{lll}
1 & 0 & 0 \\
0 & 1 & 0 \\
0 & 0 & 1
\end{array}\right], \quad \overline{\overline{\mathbf{D}}}_{3}=\frac{1}{\sqrt{3}}\left[\begin{array}{ccc}
1 & -1 & -1 \\
1 & -1 & 1 \\
1 & 1 & -1 \\
1 & 1 & 1
\end{array}\right] .
$$

We also introduce the three-dimensional extended-diagonal-axes matrix $\overline{\mathbf{E}}_{3}$ defined as

$$
\overline{\mathbf{E}}_{3}=\frac{1}{\sqrt{2}}\left[\begin{array}{ccc}
0 & 1 & -1 \\
0 & 1 & 1 \\
1 & 0 & -1 \\
1 & 0 & 1 \\
1 & -1 & 0 \\
1 & 1 & 0
\end{array}\right] .
$$


a)

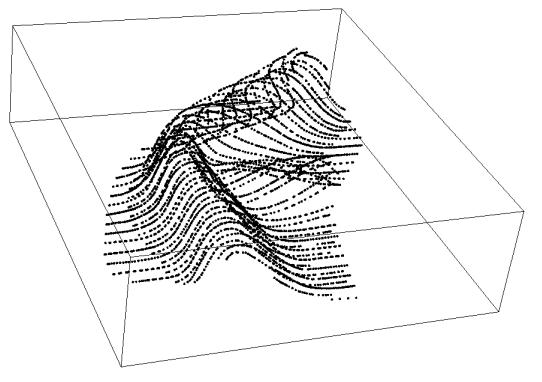

b)



c)

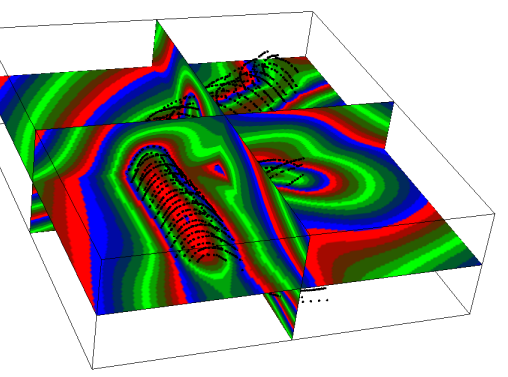

Fig. 6 Implicit stratigraphic modeling of the Balzes fold model of Ramón et al. (2015) a) Input data consisting of horizon points picked along two horizons. b) Solid view of the resulting stratigraphic field. c) Section view of the resulting stratigraphic field. Only value constraints were used in this experiment.

The three-dimensional extended-diagonal matrix $\overline{\mathbf{E}}_{3}$ is obtained by inserting an extra zero at different positions of each row of the two-dimensional diagonal matrix $\overline{\overline{\mathbf{D}}}_{2}$. The full three-dimensional direction matrix is defined as

$$
\overline{\overline{\mathbf{F}}}_{3}=\left[\begin{array}{l}
\overline{\overline{\mathbf{C}}}_{3} \\
\overline{\overline{\mathbf{D}}}_{3} \\
\overline{\mathbf{E}}_{3}
\end{array}\right]
$$

and its rows give all the directions available, locally, on a three-dimensional grid.

Following the same reasoning as in Sect. 4, we use the direction matrix $\overline{\overline{\mathbf{F}}}_{3}$ to propose the following choices for the roughness operators: 


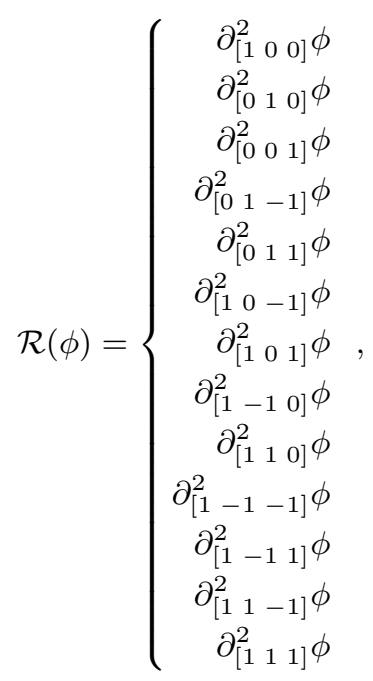

and

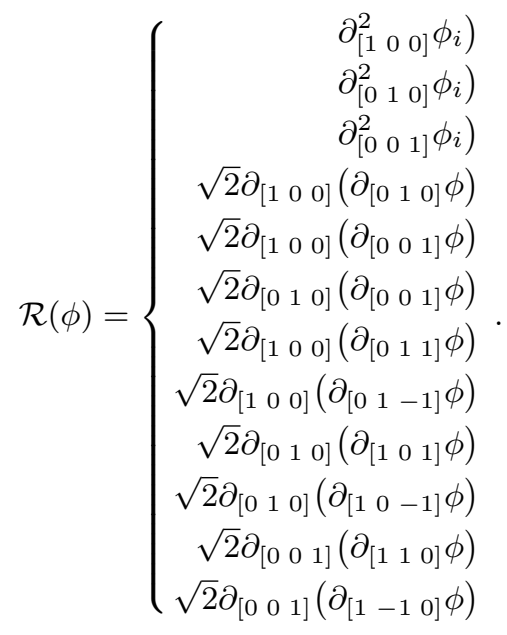

Equation (35) comes from the directional second derivative approach (Eq. (22), and Eq. (36) comes the mixed derivatives approach (Eq. (23)). It is also possible to combine these two approaches to get a third hybrid operator that satisfies the maximum-rank property (Sect. 3.2); in particular 


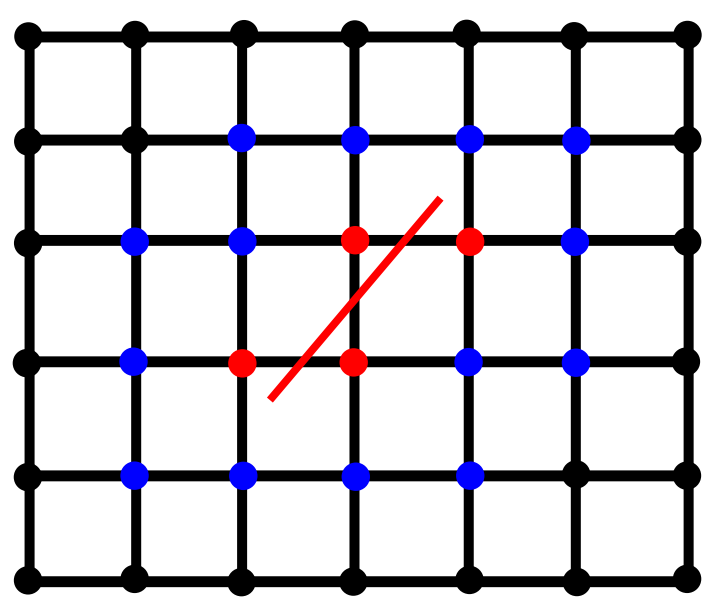

Fig. 7 Domain discretization in the presence of a discontinuity. The red line and nodes represent a discontinuity and its rasterization. Red nodes are tagged as external nodes. Blue nodes are boundary nodes, and black nodes are internal nodes.

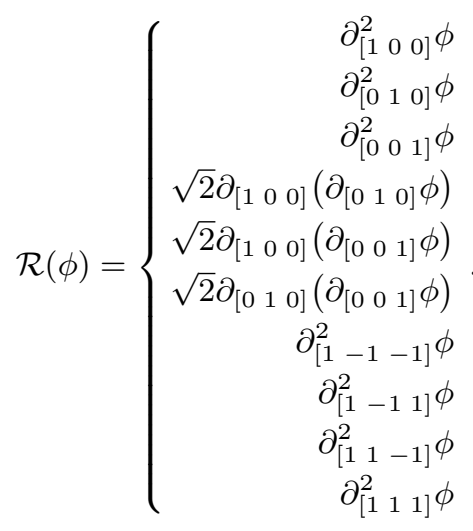

The analytic expressions of each term in these operators are given in the Appendix. While these operators may look complex as we increase dimensions, their discretizations remain straightforward: all the second derivative terms are discretized using Eq. (14), and all the mixed derivatives terms are discretized using Eq. (15). This means that the operator in Eq. (35) is implemented exactly as its two-dimensional (Eq. (26)), and its one-dimensional (Eq. (20) version. The only thing that changes is the number of directional vectors and their dimension. Figure 6 shows an example using this smoothing operator to the Balzes model of Ramón et al. (2015).

\section{Handling discontinuities}

Discontinuities are often encountered in geological structural modeling. They usually consist of faults, unconformities and intrusive bodies. We propose a simple 
a)

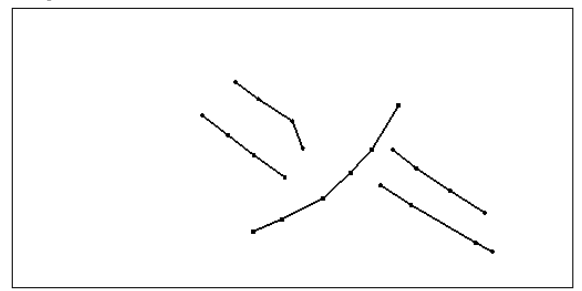

b)

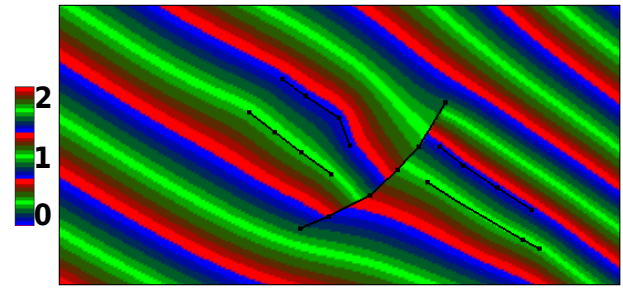

Fig. 8 Implicit stratigraphic modeling of model in Figure 5 in the presence a discontinuity. a) Input data, two horizons and one fault. b) Resulting stratigraphic field. Only value constraints were used in this experiment.

way to handle discontinuities: grid points affected by a discontinuity, after its rasterization, are tagged as external grid points (grid points in $\Omega_{E}$ ). Consider for example the illustration in Fig. 77 the discontinuity represented by the red line is rasterized to obtain the red external grid points, then boundary grid points are obtained from the definition in Eq. (8). In fact, handling discontinuities in this manner was the main motivation for most of the formalism presented earlier such as the definitions in Eqs. (6), (7), (8), and the maximum-rank property of Sect. 3.2. This formalism resolves some issues raised by Mallet (1989); Mallet (1989) cautioned that the discretization of the roughness operator $\mathcal{R}(\phi)$ may require special attention at model boundaries and near discontinuities. No special treatment is required at boundaries here, since the roughness operator is only discretized on internal grid points. For the illustration in Fig. 77, the solution would be obtained everywhere except at the red grid points, which belong to $\Omega_{E}$. Values at the red grid points may be estimated by extrapolation as a post-processing step. As an example, tagging grid points rasterized by the fault in Fig. 8. a as points in $\Omega_{E}$, changes the stratigraphic field from Fig. 5 b to the stratigraphic field in Fig. 8.b. This straightforward approach has proven capable of handling quite complex fault networks as shown in Fig. 9. It also generalizes to higher dimensions as demonstrated by the three-dimensional example in Fig. 10.

\section{Limitations and discussion}

\subsection{Large thickness variations}

When a layer exhibits large thickness variations, the resulting implicit field can have strong artifacts. For example, Fig. 11 a shows an implicit function obtained using Eq. 27) when stratigraphic layers have strong thickness variations; the resulting function contains loops, which is physically impossible from a stratigraphic modeling point of view. A more stratigraphically admissible implicit function is shown in Fig. 11. Handling such large thickness variations automatically, as done in Fig. 11, is still an active area of research (Laurent, 2016, Renaudeau et al. 2017b); we defer that discussion for further investigation. The problem highlighted by Fig. 11 a is common to most implicit modeling techniques. According to Smith and Wessel (1990), this problem seems to come from our need to look for functions with continuous second derivatives everywhere. 
a)

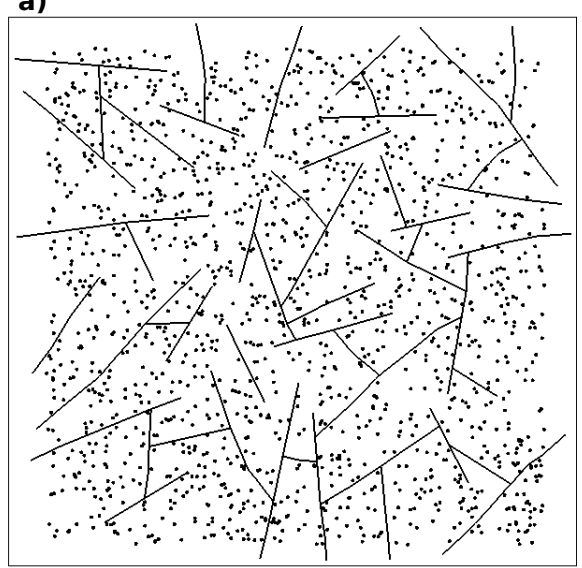

b)

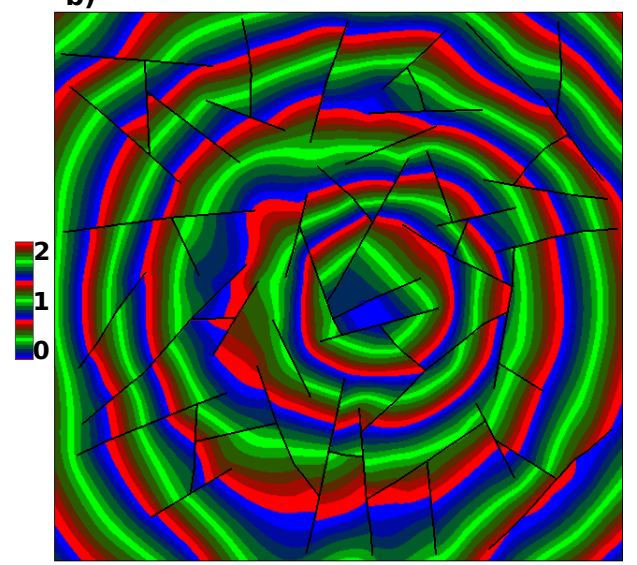

Fig. 9 Implicit stratigraphic modeling in a highly faulted model. a) Input data, 1400 data points and 50 faults. b) Resulting stratigraphic field. Only value constraints were used in this experiment. Synthetic data courtesy of ExxonMobil.

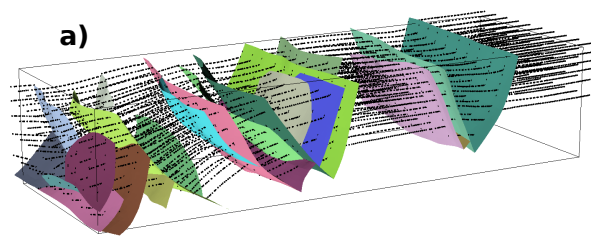

b)

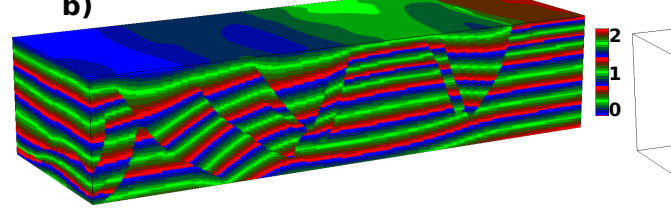

c)

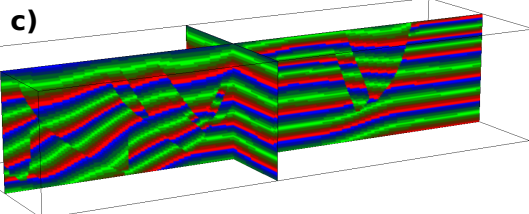

Fig. 10 Implicit stratigraphic modeling of the three-dimensional sandbox model of Chauvin et al. (2018). a) Input data consisting of 27 fault surfaces and horizon points picked along 6 horizons. b) Solid view of the resulting stratigraphic field. c) Section view of the resulting stratigraphic field. Only value constraints were used in this experiment. Initial CT data courtesy of IFPEN and C\&C Reservoirs.

\subsection{Discontinuities}

The method proposed to handle discontinuities in Sect. 6 can be numerically inefficient in some cases. If two faults are very close to one another, and there are some data between them, we are forced to use a fine grid in order to take into account those points. This can dramatically increase both the computational time and the computer memory required to solve the system depending on how close the faults are. One strategy to address this resolution limitation in finite difference implicit modeling would be to use additional degrees of freedoms on nodes adjacent to faults as done for example in the extended finite element method (Moes 


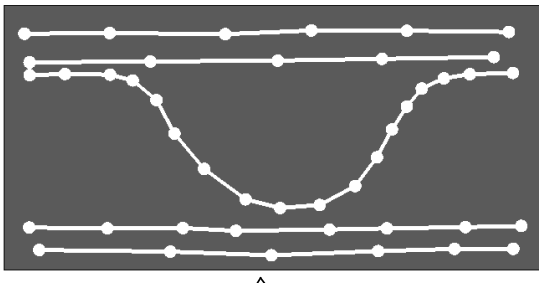

a)

\section{b)}
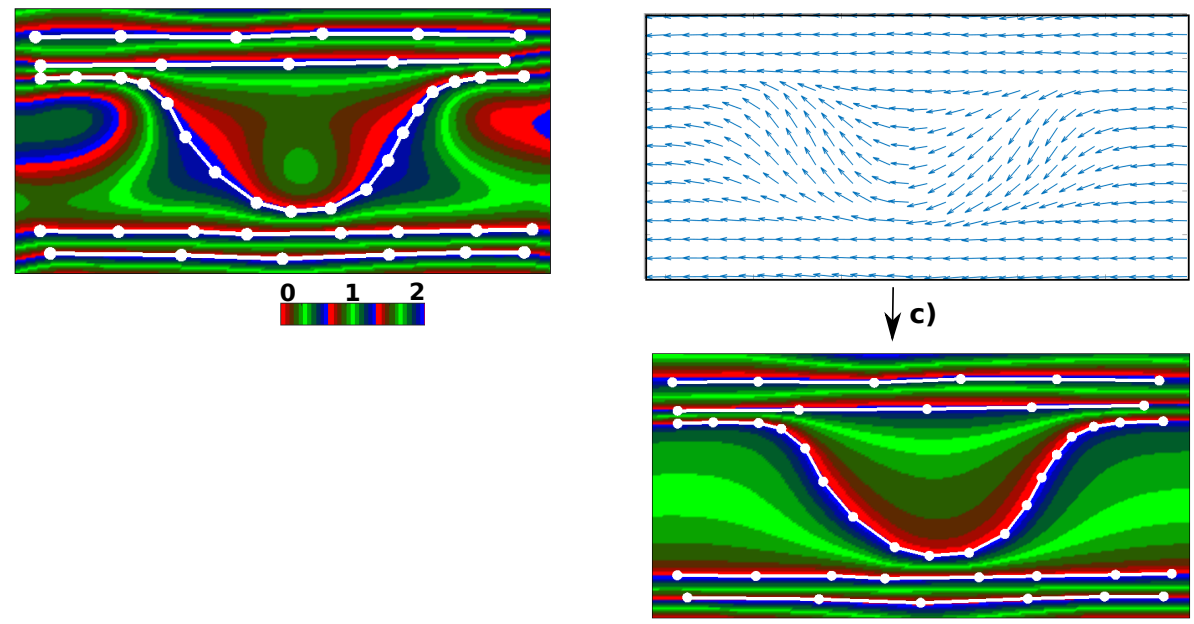

Fig. 11 Implicit modeling of the large thickness variation benchmark model of Renaudeau et al. (2017b). a) Resulting implicit function using value constraints. b)Estimation of stratigraphic orientation vectors. c) Resulting implicit function using value and orientation constraints.

and Belytscheko, 2002), and also recently used in structural modeling (Renaudeau et al., 2018).

\subsection{Performance}

We use a multi-grid conjugate gradient solver running on GPU on a computer equipped with a $3.5 \mathrm{GHz}$ core and a Quadro M4000 GPU. The two-dimensional problem takes about a second for a problem with $250 \mathrm{~K}$ grid points, and $3-4$ seconds for a problem with $1 \mathrm{M}$ grid points. The three-dimensional problem takes about 10-12 seconds for a problem with $1 \mathrm{M}$ grid points, and about 50 seconds for a problem with $5 \mathrm{M}$ grid points. Fig. 12 suggests that the running time is a quasi-linear function of the grid gize. Future research may include trying to improve performance by preconditioning our solver; some preconditioners for such a problem can be found in Wu and Hale (2015). One limitation of the proposed method is the high memory requirement for three-dimensional problems; if the first roughness 




Fig. 12 Interpolation of a complex surface using value constraints and gradient constraints (i.e. $\nabla \phi=\frac{\mathbf{g}}{\|\mathbf{g}\|}$, for a gradient vector $\mathbf{g}$ ). a) Input data: 3400 points (black) and 675 gradient vectors (red). b) Resulting stratigraphic field. c) The running time is a quasi-linear function of grid size.

operator from Eq. 35 is used, each grid point has a memory cost of at least

$$
\begin{aligned}
& 13 \text { equations } \times 3 \text { nonzeros } \\
& \times(1 \text { row id }+1 \text { column id }+1 \text { value }) \\
& \times 4 \text { bytes }=468 \text { bytes. }
\end{aligned}
$$

\subsection{Beyond three-dimensions}

Sections 4 and 5 gave particular applications of Eqs. 22] and (23) in two and three-dimensions. While the formulas for the roughness operator $\mathcal{R}(\phi)$ appeared to become more complex as we went from one, to two, to three-dimensions, the discrete version of the roughness operator did not change. The only change was in the size of the full directional matrix $\overline{\overline{\mathbf{F}}}$. It is reasonable to expect that this remains true for all higher dimensions $\mathrm{N}>3$. That is, all we need for an $\mathrm{N}$-dimensional interpolation problem is to determine the $\mathrm{N}$-dimensional directional matrix $\overline{\overline{\mathbf{F}}}_{\mathrm{N}}$. In general, the directional matrix is given by

$$
\overline{\overline{\mathbf{F}}}_{\mathrm{N}}=\left[\begin{array}{c}
\overline{\overline{\mathbf{C}}}_{\mathrm{N}} \\
\overline{\overline{\mathbf{D}}}_{\mathrm{N}} \\
\overline{\overline{\mathbf{E}}}_{\mathrm{N}}
\end{array}\right],
$$

with the understanding that the diagonal-axis matrix $\overline{\overline{\mathbf{D}}}_{\mathrm{N}}$ only exists for $\mathrm{N}>1$, and the extented-diagonal-axis matrix $\overline{\mathbf{E}}_{\mathrm{N}}$ only exists for $\mathrm{N}>2$. The Cartesianaxis and diagonal-axis matrices are given by

$$
\begin{aligned}
& \overline{\overline{\mathbf{C}}}_{\mathrm{N}}(i, j)=\delta_{i j} . \\
& \overline{\overline{\mathbf{D}}}_{\mathrm{N}}(i, j)=(-1)^{\mathrm{f}(i, j)}, \text { with } \mathrm{f}(i, j)=(2 \cdot i)^{(j+1-\mathrm{N})} .
\end{aligned}
$$


The extended-diagonal-axis matrix $\overline{\mathbf{E}}_{\mathrm{N}}$ is obtained by inserting a zero at different positions of each row of the matrix

$$
\left[\begin{array}{l}
\overline{\overline{\mathbf{D}}}_{\mathrm{N}-1} \\
\overline{\overline{\mathbf{E}}}_{\mathrm{N}-1}
\end{array}\right]
$$

in a similar way that we obtained $\overline{\mathbf{E}}_{3}$ from $\overline{\bar{D}}_{2}$ in Section $5 \overline{\overline{\mathbf{F}}}_{\mathrm{N}}$ has $\frac{3^{\mathrm{N}}-1}{2}$ rows and $\mathrm{N}$ columns. 


\section{Conclusion}

We have introduced a new technique for implicit modeling on Cartesian grids. We identified inherent practical properties of the well behaved one-dimensional discrete second derivative operator classically used to regularize interpolation in one-dimension, and then we designed higher dimensional discrete regularization operators with the same properties. In doing so, we obtained regularization operators that discretize naturally on the Cartesian grid: the operators do not require special treatment on boundary nodes, and they generalize to higher dimensions easily. Discarding boundary conditions allowed us to handle discontinuities by simply surrounding them with boundary nodes. As a result, our method is easy to implement, even in the presence of discontinuities. Numerical experiments demonstrate the robustness and numerical efficiency of the proposed method.

Acknowledgements The authors would like to thank Total for the data used in Fig. 1 , Hao Huang (ExxonMobil) for the data used in Fig. 9 and Benjamin Chauvin (previously RING, currently Harvard), IFPEN, C\&C Reservoirs for the data used in Fig. 10 Modeste Irakarama would also like to thank Paul Cupillard (RING) and Pierre Thore (Total) for their encouragement to publish this work. We are very thankful to three anonymous reviewers for the time they dedicated to review this paper. This work was done in the frame of the RING project at Université de Lorraine. We would therefore like to thank the sponsors of the RINGGOCAD Consortium managed by ASGA for their support. Software corresponding to this paper is available to consortium members in the SIGMA package.

\section{Conflict of interest}

The authors declare that they have no conflict of interest. 


\section{Appendix}

The analytic expressions of terms appearing in the formulas for $\mathcal{R}(\phi)$, as proposed in Sect. 5, are given below:

$$
\begin{aligned}
& \partial_{\left[\begin{array}{lll}
1 & 0 & 0
\end{array}\right]}^{2} \phi=\partial_{x}^{2} \phi,
\end{aligned}
$$

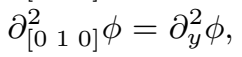

$$
\begin{aligned}
& \partial_{\left[\begin{array}{lll}
0 & 0 & 1
\end{array}\right]}^{2} \phi=\partial_{z}^{2} \phi, \\
& \sqrt{2} \partial_{\left[\begin{array}{lll}
1 & 0 & 0
\end{array}\right]}\left(\partial_{\left[\begin{array}{lll}
0 & 1 & 0
\end{array}\right]} \phi\right)=\sqrt{2} \partial_{x}\left(\partial_{y} \phi\right), \\
& \sqrt{2} \partial_{\left[\begin{array}{lll}
1 & 0 & 0
\end{array}\right]}\left(\partial_{\left[\begin{array}{lll}
0 & 0 & 1
\end{array}\right]} \phi\right)=\sqrt{2} \partial_{x}\left(\partial_{z} \phi\right), \\
& \sqrt{2} \partial_{\left[\begin{array}{lll}
1 & 1 & 0]
\end{array}\right]}\left(\partial_{\left[\begin{array}{lll}
0 & 0 & 1
\end{array}\right]} \phi\right)=\sqrt{2} \partial_{y}\left(\partial_{z} \phi\right), \\
& \sqrt{2} \partial_{\left[\begin{array}{lll}
1 & 0 & 0
\end{array}\right]}\left(\partial_{\left[\begin{array}{lll}
0 & 1 & 1
\end{array}\right]} \phi\right)=\partial_{x}\left(\partial_{y} \phi+\partial_{z} \phi\right) \text {, } \\
& \sqrt{2} \partial_{\left[\begin{array}{lll}
1 & 0 & 0
\end{array}\right]}\left(\partial_{\left[\begin{array}{lll}
0 & 1 & -1
\end{array}\right]} \phi\right)=\partial_{x}\left(\partial_{y} \phi-\partial_{z} \phi\right),
\end{aligned}
$$

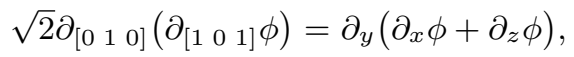

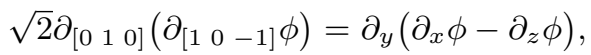

$$
\begin{aligned}
& \sqrt{2} \partial_{\left[\begin{array}{lll}
0 & 0 & 1
\end{array}\right]}\left(\partial_{\left[\begin{array}{lll}
1 & 1 & 0
\end{array}\right]} \phi\right)=\partial_{z}\left(\partial_{x} \phi+\partial_{y} \phi\right) \text {, } \\
& \sqrt{2} \partial_{\left[\begin{array}{lll}
0 & 1
\end{array}\right]}\left(\partial_{\left[\begin{array}{lll}
1-1 & 0
\end{array}\right]} \phi\right)=\partial_{z}\left(\partial_{x} \phi-\partial_{y} \phi\right) \text {, } \\
& \partial_{\left[\begin{array}{ll}
0 & 1-1]
\end{array} \phi\right.}^{2} \phi=\frac{1}{2}\left(\partial_{y}^{2}+\partial_{z}^{2}-2 \partial_{y} \partial_{z}\right) \phi, \\
& \partial_{[011]}^{2} \phi=\frac{1}{2}\left(\partial_{y}^{2}+\partial_{z}^{2}+2 \partial_{y} \partial_{z}\right) \phi \\
& \partial_{\left[\begin{array}{ll}
1 & 0-1]
\end{array}\right]}^{2} \phi=\frac{1}{2}\left(\partial_{x}^{2}+\partial_{z}^{2}-2 \partial_{x} \partial_{z}\right) \phi \text {, } \\
& \partial_{\left[\begin{array}{lll}
1 & 01
\end{array}\right]}^{2} \phi=\frac{1}{2}\left(\partial_{x}^{2}+\partial_{z}^{2}+2 \partial_{x} \partial_{z}\right) \phi, \\
& \partial_{[1-10]}^{2} \phi=\frac{1}{2}\left(\partial_{x}^{2}+\partial_{y}^{2}-2 \partial_{x} \partial_{y}\right) \phi, \\
& \partial_{\left[\begin{array}{ll}
1 & 10]
\end{array} \phi\right.}^{2} \phi=\frac{1}{2}\left(\partial_{x}^{2}+\partial_{y}^{2}+2 \partial_{x} \partial_{y}\right) \phi \text {, } \\
& \partial_{[1-1-1]}^{2} \phi=\frac{1}{3}\left(\partial_{x}^{2}+\partial_{y}^{2}+\partial_{z}^{2}-2 \partial_{x} \partial_{y}-2 \partial_{x} \partial_{z}+2 \partial_{y} \partial_{z}\right) \phi \text {, } \\
& \partial_{[1-11]}^{2} \phi=\frac{1}{3}\left(\partial_{x}^{2}+\partial_{y}^{2}+\partial_{z}^{2}-2 \partial_{x} \partial_{y}+2 \partial_{x} \partial_{z}-2 \partial_{y} \partial_{z}\right) \phi \text {, }
\end{aligned}
$$

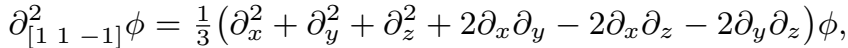

$$
\begin{aligned}
& \partial_{[111]}^{2} \phi=\frac{1}{3}\left(\partial_{x}^{2}+\partial_{y}^{2}+\partial_{z}^{2}+2 \partial_{x} \partial_{y}+2 \partial_{x} \partial_{z}+2 \partial_{y} \partial_{z}\right) \phi .
\end{aligned}
$$




\section{References}

Ian Briggs. Machine countouring using minimum curvature. Geophysics, 39(1): 39-48, 1974.

P Calcagno, J.P. Chilès, G. Courrioux, and A. Guillen. Geological modelling from the field data and geological knowledge Part I. Modeling method coupling 3D potential-field interpolation and geological rules. Physics of the Earth and Planetary Interiors, 17:147-157, 2008.

G. Caumon, P. Collon, C. Le Carlier de Veslud, S. Viseur, and J. Sausse. SurfaceBased 3D Modeling of Geological Structures. Mathematical Geosciences, 41: 927-945, 2009.

Guillaume Caumon. Towards stochastic time-varying geological modeling. Mathematical Geosciences, 42:555-569, 2010.

Guillaume Caumon, Gary Gray, Christophe Antoine, and Marc-Olivier Titeux. Three-dimensional implicit stratigraphic model building from remote sensing data on tetrahedral meshes: theory and application to regional model of La Popa Basin, NE Mexico. IEEE transactions on geoscience and remote sensing, 51(3), 2013.

H. Chauris, M. Noble, G. Lambaré, and P. Podvin. Migration velocity analysis from locally coherent events in 2-D laterally heterogeneous media, Part I: Theoretical aspects. Geophysics, 67(4), 2002.

Benjamin Chauvin, Peter Lovely, Joseph Stockmeyer, Andreas Plesch, Guillaume Caumon, and John Shaw. Validating novel boundary conditions for threedimensional mechanics-based restoration: An extensional sandbox model example. AAPG Bulletin, 102:245-266, 2018.

Nicolas Cherpeau and Guillaume Caumon. Stochastic structural modelling in sparse data situations. Petroleum Geoscience, 21:233-247, 2015.

J-P. Chilès, C. Aug, A. Guillen, and T. Less. Modeling the geometry of geological units and its uncertainty in $3 \mathrm{~d}$ form structural data: the potential-field method. In Orebody Modeling and Strategic Mine Planning, volume Spectrum 14, Perth, WA, 2004.

E.J. Cowan, R.K. Beatson, W.R. Fright, T.J. McLennan, and T.J. Mitchell. Rapid geological modeling. In Applied Structural Geology for Mineral Exploration and Mining, Kalgoorlie, Australia, September 2002.

José Enriquez, Jean Thomann, and Michel Goupillot. Applications of bidimensional spline functions to geophysics. Geophysics, 48(9):1268-1273, 1983.

Gijs Fehmers and Christian Höcker. Fast structural intepretation with structureoriented filtering. Geophysics, 68(4), 2003.

Tobias Frank. 3D reconstruction of complex geological interfaces from irregularly distributed and noisy point data. Computers and Geosciences, 33:932-943, 2007.

Gabriel Godefroy, Francois Bonneau, Guillaume Caumon, and Gautier Laurent. Stochastic association of fault evidences using graph theory and geological rules. In 2017 RING meeting, Université de Lorraine-ENSG, Nancy, France, September 2017. ASGA.

Italo Gomes Gonçalves, Sissa Kumaira, and Felipe Guadagnin. A machine learning approach to the potential-field method for implicit modeling of geological structures. Computers and Geosciences, 103:173-182, 2017.

Lachlan Grose, Gautier Laurent, Laurent Ailleres, Robin Armit, Mark Jessell, and Guillaume Caumon. Structural data constraints for implicit modeling of folds. 
Journal of Structural Geology, 104:80-92, 2017.

Michael Hillier, Ernst Schetselaar, Eric Kemp, and Gervais Perron. ThreeDimensional Modeling of Geological Surfaces Using Generelized Interpolation with Radial Basis Functions. Mathematical Geosciences, 46:931-953, 2014.

Mark Jessell, Laurent Ailleres, Eric de Kemp, Mark Lindsay, Florian Wellmann, Michael Hillier, Gautier Laurent, Thomas Carmichael, and Roland Martin. Next generation three-dimensional geologic modeling and inversion. Society of Economic Geologists Special Publication, 18:261-272, 2014.

Christian Lajaunie, Gabriel Courrioux, and Laurent Manuel. Foliation fields and $3 \mathrm{D}$ cartography in geology: Principles of a method based on potential interpolation. Mathematical Geology, 29(4), 1997.

Gautier Laurent. Iterative thickness regularization of stratigraphic layers in discrete implicit modeling. Mathematical Geosciences, 48:811-833, 2016.

Gautier Laurent, Laurent Ailleres, Lachlan Grose, Guillaume Caumon, Mark Jessell, and Robin Armit. Implicit modeling of folds and overprinting deformation. Earth and Planetary Science Letters, 456:26-38, 2016.

David Ledez. Modélisation d'objets naturels par formulation implicite. PhD thesis, Université de Lorraine-ENSG, Nancy, France, 2003.

Bruno Levy. Topologie Algorithmique, Combinatoire et Plongement. PhD thesis, Université de Lorraine-ENSG, Nancy, France, 1999.

Jean-Laurent Mallet. Discrete smooth interpolation. ACM Transactions on Graphics, 8:121-144, 1989.

Jean-Laurent Mallet. Discrete smooth interpolation in geometric modeling. Computer-Aided Design, 24:178-191, 1992.

Jean-Laurent Mallet. Discrete Modeling for Natural Objects. Mathematical Geology, 29(1), 1997.

Jean-Laurent Mallet. Space-time mathematical framework for sedimentary geology. Mathematical Geology, 36(1), 2004.

Jean-Laurent Mallet. Elements of mathematical sedimentary geology: The GeoChron model. EAGE, 2014.

J.L. Mallet. Automatic contouring in presence of discontinuities. In G. Verly, M. David, A. G. Journel, and M. Marechal, editors, Geostatistics for natural resources characterization, Part 2, pages 669-677. D. Reidel Publishing Company, 1984.

Ryan Martin and Jeff Boisvert. Iterative refinement of implicit boundary models for improved geological feature reproduction. Computers and Geosciences, 109: $1-15,2017$.

Nicolas Moes and Ted Belytscheko. Extended finite element for cohesive crack growth. Engineering Fracture Mechanics, 69:813-833, 2002.

Rémi Moyen. "Paramétrisation 3D de l'espace en géologie sédimentaire: le modèle GeoChron". PhD thesis, Université de Lorraine-ENSG, Nancy, France, 2005.

Timothy Newman and Hong Yi. A survey of the marching cubes algorithm. Coomputers \&6 Graphics, 30:854-879, 2006.

Maria José Ramón, Emilio L. Pueyo, Guillaume Caumon, and José Luis Briz. Parametric unfolding of flexural folds using palaeomagnetic vectors. Geological Society of London, 425:247-258, 2015.

J. Renaudeau, M. Irakarama, G. Laurent, F. Maerten, and G. Caumon. Implicit modeling of geological structures: A Cartesian grid method handling discontinuities with ghost points. In 41st International Conference on Boundary Elements 
and other Mesh Reduction Methods, New Forest, UK, 2018.

Julien Renaudeau, Emmanuel Malvesin, Frantz Maerten, and Guillaume Caumon. Towards continuous equations behind implicit stratigraphic modeling. In 2017 RING meeting, Université de Lorraine-ENSG, Nancy, France, September 2017a. ASGA.

Julien Renaudeau, Emmanuel Malvesin, Frantz Maerten, and Guillaume Caumon. The weighted minimization criterion as a physical way to handle thickness variation in implicit structural modeling. In 2017 RING meeting, Université de Lorraine-ENSG, Nancy, France, September 2017b. ASGA.

Julien Renaudeau, Emmanuel Malvesin, Frantz Maerten, and Guillaume Caumon. Implicit Structural Modeling by Minimization of the Bending Energy with Moving Least Squares Functions. Mathematical Geosciences, 51:693-724, 2019.

W. Smith and P. Wessel. Gridding with continous curvature splines in tension. Geophysics, 55(3):293-305, 1990.

L. Souche, F. Lepage, and G. Iskenova. Volume Based Modeling - Automated construction of complex structural models. In 75th EAGE Conference and Exhibition, 2013.

Anne-Laure Tertois. "Création et modification de modèles géologiques par champs de potentiel. Application au modèle GeoChron". PhD thesis, Université de Lorraine-ENSG, Nancy, France, 2007.

Greg Turk and James O'Brien. Modelling with Implicit Surfaces that Interpolate. ACM Transactions on Graphics, 21:855-873, 2002.

Paul Wessel and David Bercovici. Interpolation with splines in tension: a Green's function approach. Mathematical Geology, 30(1), 1998.

Xinming Wu. Building 3D subsurface models conforming to seismic structural and stratigraphic features. Geophysics, 82:IM21-IM30, 2017.

Xinming $\mathrm{Wu}$ and Dave Hale. Horizon volumes with interpreted constraints. Geophysics, 80:IM21-IM33, 2015. 